\title{
Loneliness, Friendship, and Facebook Intrusion. A Study in Poland, Slovakia, Syria, Malaysia, and Ecuador
}

\author{
Agata Błachnio, Aneta Przepiórka, Mariusz Wołońciej \\ Institute of Psychology, The John Paul II Catholic University of Lublin, \\ Poland
}

\author{
Ali Bassam Mahmoud \\ University of Liverpool, United Kingdom
}

Juraj Holdoš

Department of Psychology, Catholic University in Ružomberok, Slovak Republic

\section{Eiad Yafi}

Universiti Kuala Lumpur, Malaysia

\begin{abstract}
With the increasing popularity of Facebook, a new social phenomenon connected with its extensive use has appeared: namely, Facebook intrusion. The main aim of the study was to examine the mediating role of loneliness in the relation between friendships and Facebook intrusion. We analyzed data from five countries: Poland $(N=399)$, Slovakia $(N=266)$, Syria $(N=475)$, Ecuador $(N=327)$, and Malaysia $(N=241)$. The total sample consisted of 1731 participants who had Facebook accounts; $61 \%$ of the participants were women. We administered the Facebook Intrusion Scale, the Loneliness Scale, and the Friendship Scale. The results indicated differences in the role of loneliness and friendship in Facebook intrusion, depending on the country. Among young Polish people, friendship decreases loneliness and loneliness decreases Facebook intrusion. Whereas among Ecuadorians friendship decreases loneliness, while loneliness increases Facebook intrusion.
\end{abstract}

Key words: Facebook intrusion, loneliness, friendship, culture

Facebook is one of the most popular social networking sites. As shown in research conducted by the Pew Research Center (2014), time spent on Facebook becomes longer every year. In a sample of 1597 users, $70 \%$ used Facebook

Acknowledgement

This research was supported by a grant from the National Science Centre (NCN) No. 2014/15/B/HS6/ 03129

Correspondence concerning this article should be addressed to Agata Błachnio, Institute of Psychology, The John Paul II Catholic University of Lublin, Poland, Al. Racławickie 14, 20-950 Lublin, Poland. E-mail: gatta@kul.pl

Received January 15, 2018 several times a day. The site is extremely popular, especially with the young generation, whose members use e-mail or text messages less and less often and increasingly often use a Facebook account. The terms interchangeably used in the literature in reference to Facebook dependency are "Facebook intrusion" (Elphinston \& Noller, 2011) and "Facebook addiction" (Andreassen \& Pallesen, 2014); the phenomenon they refer to can be defined as excessive involvement in Facebook, disrupting day-to-day activities and interpersonal relationships (Elphinston \& Noller, 2011). The authors mention three aspects of this phenomenon, namely: 1) withdrawal, 2) relapse and reinstatement, and 3) euphoria. People who are strongly involved in Facebook 
feel distress when they cannot stop using it. What is more, they have unsuccessfully attempted to reduce Facebook use. They also feel a connection with other people who use Facebook (Elphinston \& Noller, 2011).

On the basis of the literature, it can be said that there are certain psychological characteristics and resources that predispose individuals to excessive use both of the Internet and Facebook. A number of studies mention loneliness as one of these characteristics. Loneliness occurs when a difference is perceived between the desired and actual levels of social involvement (Russell, Cutrona, McRae, \& Gomez, 2012). For instance, a high level of social loneliness increases the risk of pathological Internet use (Wegmann \& Brand, 2016). Others suggest that loneliness can predict Internet addiction (Özdemir, Kuzucu, \& Ak, 2014). Still another study showed that the link between technology use and physical and psychological health was mediated by reduced loneliness (Chopik, 2016). Loneliness has also been found to correlate positively with passive engagement on Facebook (e.g., groups or fan pages; Ryan \& Xenos, 2011).

Some results suggest the role of loneliness in using Facebook excessively. The more time people spend on Facebook, the more lonely they feel; however, if they use both Facebook and Twitter rather than only Facebook, they feel less lonely (Petrocchi, Asnaani, Martinez, Nadkarni, \& Hofmann, 2015). Some authors assumed that loneliness would turn out to be an important determinant of social media use (Ye \& Lin, 2015; Song et al., 2014); . Loneliness has been found to be a positive predictor of Facebook addiction, standard Facebook use, and Facebook entertainment (Błachnio et al., 2016b). Scholars also found that the relationship between the need for privacy and excessive Facebook use was negative and significant when loneliness was introduced as a mediator. It is therefore possible to speak of a para- doxical effect of the need for privacy. On the one hand, the need for privacy can protect a person from excessive Facebook use, but on the other hand, a high level of the need for privacy is associated with loneliness, and lonely people are more prone to excessive Facebook use (Błachnio, Przepiorka, Bałakier, \& Boruch, 2016). Friendship and loneliness are only partially the same concept. A person can be socially isolated (i.e., he or she may not have relationships with others) without feeling lonely (Hawthorne \& Griffith, 2000). Some results indicate that Facebook dependency is related to relationship dissatisfaction (Elphinston \& Noller, 2011). Biolcati and Cani (2015) explored friendship and loneliness in relation to Facebook use. They found that those who felt lonely were more dissatisfied with their online and offline relationships: these people met friends in person less frequently and considered their online contacts less satisfactory. In addition, MorahanMartin and Schumacher (2003) found that lonely people may search for online contacts, as Internet facilitates social behaviors, and that they may make friendships online, as anonymity and the lack of physical presence may be attractive.

Some evidence suggests that there are cultural factors involved in the Facebook intrusion phenomenon (Błachnio et al., 2016). The authors showed that variables differentiating cultures, such as uniqueness, were negatively and low context positively related to Facebook intrusion (Błachnio, Przepiorka, Benvenuti et al., 2016). A great body of studies highlighted different patterns in Internet use (Gong Li \& Stump, 2007; Singh, Fassot, Chao, \& Hoffmann, 2006) and Facebook use (Brailovskaia \& Bierhoff, 2016; Peters, Winschiers-Theophilus, \& Mennecke, 2015; Vasalou et al., 2010) across various countries. Some differences have also been found in the relations between loneliness, the sense of social capital, and SNS use among three countries: South Korea, China, and the 
USA (Ji et al., 2010). US users mostly were focused on exchanging and sharing opinions and information, while Korean and Chinese users tended to focus on the emotional climate of communication.

Our study was conducted in five countries: Poland, Slovakia, Syria, Ecuador, and Malaysia. These countries were selected because they differ in terms of political, industrial, economic, societal, and technological development. First of all, Internet penetration rates differ across these countries (statistics from 2017) ${ }^{1,2}$, the figures being 78.1 in Poland, 85.0 in Slovakia, 33.0 in Syria, 79.9 in Ecuador, and 78.3 in Malaysia. These discrepancies in access to the Internet and Facebook result in different backgrounds for their users. Internet penetration expresses the relationship between the number of Internet users in each country and its demographic data (Calderaro, 2010). The relationship between Facebook intrusion and Internet penetration was demonstrated in the previous studies (Błachnio, Przepiorka, Benvenuti, Cannata, Ciobanu, Senol-Durak et al., 2016a). More differences between the countries included in our study are shown in the Inglehart-Welzel cultural map of the world ${ }^{3}$, where countries are arranged according to two dimensions: traditional vs. secular-rational values and survival vs. selfexpression values. Apart from that, they differ in terms of the World Happiness Index that ranks countries by their happiness level ${ }^{4}: 6.123$ for Poland, 5.747 for Slovakia, 3.516 for Syria, 6.322 for Malaysia, and 5.973 for Ecuador. The plethora of research showed the importance of happiness in the context of Facebook use (e.g., Satici \& Uysal, 2015).

The general aim of our study was to determine the role of psychological factors, such as loneliness and friendship in the relation to

\footnotetext{
${ }^{1} \mathrm{https} / / / \mathrm{www}$. internetworldstats.com

${ }^{2} \mathrm{http}: / / \mathrm{www}$.internetlivestats.com/

${ }^{3} \mathrm{http}: / / \mathrm{www}$.worldvaluessurvey.org/

${ }^{4}$ http://worldhappiness.report/
}

Facebook intrusion across five countries previously. More specifically, we wanted to test the mediating role of loneliness in the relation between friendships and Facebook intrusion in five countries. Loneliness and friendship as they both belong to the social functioning (for a review Bukowski, Laursen, \& Rubin, 2009) seem to be the important variables in Facebook intrusion. Although the issue of loneliness and friendship has been addressed in previous studies (e.g., Petrocchi, Asnaani, Martinez, Nadkarni, \& Hofmann, 2015; Biolcati \& Cani, 2015), the cross-cultural aspect of these relations in the context of Facebook intrusion is rather new and to the best of our knowledge it was not examined previously. We would also like to compare the level of Facebook usage: log hours on weekdays and weekends within countries, as these variables were important in previous studies (e.g. Błachnio, Przepiorka, \& Hawi, 2016). The following main hypothesis was formulated: Loneliness is a mediator of the relation between friendship and Facebook intrusion. The age variable was controlled for. We also examined the differences in the number of Facebook log hours on weekdays and weekends between these countries.

\section{Method}

\section{Participants}

The participants were 1,731 individuals $(61 \%$ were women) possessing Facebook accounts, aged between 16 and 67, with a mean age of $M=23.04$ years. This included 399 Polish participants aged 16 to $67(M=21.85, S D=4.81$, $76 \%$ were female), 266 Slovakians aged 16-59 ( $M=24.64, S D=7.19,75 \%$ were female $), 475$ Syrians aged 16 to $55(M=23.86, S D=5.63,36 \%$ were female), 241 Malaysians aged 19 to 41 $(M=21.79, S D=2.38,43 \%$ were female $)$, and 327 Ecuadorians aged 17 to $66(M=23.04, S D=6.78$, $81 \%$ were female). 


\section{Procedure}

In each country we applied a snowball sampling procedure to recruit the participants. We prepared electronic versions of the questionnaires translated into the local languages and sent the link to the study website to undergraduate students, requesting them to post it on their Facebook walls and thus spread the message about the study among their Facebook friends. The participants received no remuneration for their participation.

\section{Measures}

The investigators administered the Facebook Intrusion Scale, the Loneliness Scale, and the Friendship Scale to the participants. All the instruments had been adapted in each country by means of the back-translation procedure, with great care taken to ensure grammatical and semantic correctness as well as readability. The questions about log hours on weekdays and weekends were also taken into consideration.

Facebook Intrusion Questionnaire, developed by Elphinston and Noller (Elphinston \& Noller, 2011), is based on behavioral addiction components and on a scale measuring phone involvement. It consists of eight items (e.g., I have been unable to reduce my Facebook use) measuring the relations between Facebook involvement tendency and eight aspects of behavioral addiction, namely: cognitive salience, behavioral salience, interpersonal conflict, conflict with other activities, euphoria, loss of control, withdrawal, as well as relapse and reinstatement. The items are rated on a 7-point Likert scale from 1 (strongly disagree) to 7 (strongly agree). Cronbach's $\alpha$ was .82 for the total sample, .85 for Poland, .77 for Slovakia, .80 for Syria, .97 for Malaysia, and .83 for Ecuador.

The De Jong Gierveld Loneliness Scale measures the sense of loneliness. The scale con- sists of 11 items (e.g., I miss having really close friends), six of them negatively and five positively formulated. The items are rated on a 5-point Likert scale $(1=$ completely disagree; $5=$ completely agree). Cronbach's $\alpha$ for the total sample was .84, and its values for specific countries were as follows: .91 for Poland, .87 for Slovakia, .83 for Syria, .69 for Malaysia, and .75 for Ecuador.

The Friendship Scale by Hawthorne and Griffith (2000), measuring level of social isolation (which means loss of relationships), consists of 5 items, (e.g., Ifound it easy to get on with other people). The items are rated on a 5-point scale, with answers ranging from $1=$ not at all to $5=$ almost always. Cronbach's $\alpha$ was .71 for the total sample, .79 for Poland, .73 for Slovakia, .63 for Syria, .57 for Malaysia, and .55 for Ecuador. In the case of the original version of the Friendship Scale, Cronbach's $\alpha$ was .76.

\section{Results}

\section{The Measurement Invariance}

We tested measurement invariance across the five countries. Five models were fitted with increasingly more constraints: Model 1: configural invariance - the same factor structure across groups. Model 2: weak invariance (metric) - the same factor structure and factor loadings across groups. Model 3: strong invariance (scalar) the same factor structure, factor loadings, and intercepts across groups. Model 4: strict invariance - the same factor structure, factor loadings, intercepts, and residual variances across groups. Model 5: the same factor structure, factor loadings, intercepts, residual variances, and means across groups. The analyses were performed by means of the lavaan package (Rosseel, 2012), running in the R environment (R Core Team, 2016).

In the next step, we tested the invariance of Friendship Scale. Models with gradually more 
constraints imposed were gradually significantly worse (i.e., Model 2 was worse than Model 1, Model 3 was worse than Model 2, etc.). The basic configural model also had a poor fit. In sum, we found no measurement invariance (Table 1). Next, we tested the invariance of the Loneliness Scale and did not find it significant (Table 2).
In the next step, the measurement invariance of the Facebook Intrusion scale was tested. The model imposing strong (scalar) invariance was barely significantly worse than the one assuming weak (metric) invariance (Table 3 ).

We did not obtain invariance for any of the scales, which means the investigated variables do not represent the same phenomenon in each

Table 1 Measurement invariance statistics for the Friendship Scale: A comparison across five countries

\begin{tabular}{lcrcccrcc}
\hline & \multicolumn{3}{c}{ Model fit } & \multicolumn{5}{c}{ Differences between models } \\
\hline Model & $d f$ & $\mathrm{chi}^{2}$ & CFI & RMSEA & $\Delta$ chi $^{2}$ & $\Delta d f$ & $\Delta$ CFI & $\begin{array}{c}p \\
\text { for difference }\end{array}$ \\
\hline 1. Configural & 25 & 325.67 & .82 & .19 & 155.34 & 16 & & \\
2. Weak & 41 & 481.01 & .74 & .18 & 426.55 & 16 & .08 & $<.001$ \\
3. Strong & 57 & 907.56 & .50 & .21 & 421.76 & 20 & .24 & $<.001$ \\
4. Strict & 77 & 1329.32 & .27 & .22 & 317.07 & 4 & .24 & $<.001$ \\
5. Means & 81 & 1646.39 & .09 & .24 & 155.34 & 16 & .18 & $<.001$ \\
\hline
\end{tabular}

Table 2 Measurement invariance statistics for the Loneliness Scale: A comparison across five countries

\begin{tabular}{lccccrrrr}
\hline & \multicolumn{9}{c}{ Model fit } & \multicolumn{5}{c}{ Differences between models } \\
\hline Model & $d f$ & chi $^{2}$ & CFI & RMSEA & $\Delta$ chi $^{2}$ & $\Delta d f$ & $\Delta$ CFI & $\begin{array}{c}p \\
\text { for difference }\end{array}$ \\
\hline 1. Configural & 175 & 1753.30 & .72 & .16 & 113.77 & 36 & & \\
2. Weak & 211 & 1867.10 & .70 & .15 & 1141.92 & 36 & .01 & $<.001$ \\
3. Strong & 247 & 3009.00 & .51 & .18 & 705.21 & 40 & .20 & $<.001$ \\
4. Strict & 287 & 3714.20 & .39 & .19 & 214.41 & 4 & .12 & $<.001$ \\
5. Means & 291 & 3928.60 & .35 & .19 & 113.77 & 36 & .04 & $<.001$ \\
\hline
\end{tabular}

Table 3 Measurement invariance statistics for the Facebook Scale: A comparison across five countries

\begin{tabular}{lcrrrrrrr}
\hline & \multicolumn{3}{c}{ Model fit } & \multicolumn{5}{c}{ Differences between models } \\
\hline Model & $d f$ & $\mathrm{chi}^{2}$ & CFI & RMSEA & $\Delta$ chi $^{2}$ & $\Delta d f$ & $\Delta$ CFI & $\begin{array}{c}p \\
\text { for difference }\end{array}$ \\
\hline 1. Configural & 100 & 363.11 & .93 & .09 & & & & \\
2. Weak & 128 & 468.40 & .91 & .09 & 105.28 & 28 & .02 & $<.001$ \\
3. Strong & 156 & 1229.19 & .73 & .14 & 760.8 & 28 & .19 & .054 \\
4. Strict & 188 & 1694.19 & .62 & .15 & 464.99 & 32 & .11 & .011 \\
5. Means & 192 & 1816.48 & .59 & .16 & 122.29 & 4 & .03 & .004 \\
\hline
\end{tabular}


country. This justifies analyzing them in different cultures.

\section{The Descriptive Statistics}

The descriptive statistics for each scale (means and standard deviations for all variables) are presented in Table 4.

Pearson's $r$ correlations between the variables in each of the five countries are presented in Table 5. Friendship was strongly negatively correlated with loneliness in all of the countries. Loneliness was positively cor- related with Facebook intrusion in Slovakia and in Ecuador. Friendship was positively correlated with Facebook intrusion in Malaysia. Only in Malaysia we obtained a weak correlation between friendship and Facebook intrusion; in the remaining included countries there was no correlation between these variables (see Table 5).

Additionally, the means and standard deviations for log hours on weekdays and weekends and the correlations between these variables and Facebook intrusion are presented in Table 6.

Table 4 Means and standard deviations for Facebook intrusion, loneliness, and friendship variables

\begin{tabular}{lccccccc}
\hline & \multicolumn{3}{c}{ Facebook intrusion } & \multicolumn{2}{c}{ Loneliness } & \multicolumn{2}{c}{ Friendship } \\
\hline Ecuador & $N$ & $M$ & $S D$ & $M$ & $S D$ & $M$ & $S D$ \\
Malaysia & 327 & 2.54 & 1.14 & 2.73 & 0.54 & 18.61 & 3.64 \\
Poland & 252 & 2.91 & 1.06 & 3.01 & 0.53 & 13.94 & 3.38 \\
Slovakia & 399 & 2.65 & 1.15 & 2.24 & 0.85 & 19.38 & 3.97 \\
Syria & 261 & 2.81 & 1.03 & 2.44 & 0.70 & 19.70 & 3.81 \\
\hline Note. ${ }^{*} p<.05 ; * * p<.01 ; * * * p<.001$ & & & & $*$ & & &
\end{tabular}

Table 5 Correlations between variables

\begin{tabular}{llll}
\hline & & 1 & 2 \\
\hline Ecuador & 1. Facebook intrusion & .001 & \\
& 2. Friendship & $.15^{* *}$ & $-.50^{* * *}$ \\
\hline 3. Loneliness & & \\
& 1. Facebook intrusion & $.16^{*}$ & $-.46^{* * *}$ \\
\hline 2. Friendship & -.09 & \\
3. Loneliness & & $-.79^{* * *}$ \\
\hline Poland & 1. Facebook intrusion & -.08 & \\
& 2. Friendship & .01 & $-.68^{* * *}$ \\
\hline 3lovakia & 1. Loneliness & & \\
& 2. Facebook intrusiondship & -.11 & \\
& 3. Loneliness & $.16^{*}$ & $-.65^{* * *}$ \\
\hline Syria & 1. Facebook intrusion & -.04 & .08 \\
\hline
\end{tabular}

Note. ${ }^{*} p<.05 ; * * p<.01 ; * * * p<.001$ 
Table 6 Means and standard deviations for log hours on weekdays and on weekends and their correlations with Facebook intrusion

\begin{tabular}{lccccccc}
\hline & \multicolumn{2}{c}{$\begin{array}{c}\text { log hours on } \\
\text { weekdays }\end{array}$} & $\begin{array}{c}\text { correlations } \\
\text { with Facebook } \\
\text { intrusion }\end{array}$ & $\begin{array}{c}\text { log hours on } \\
\text { weekends }\end{array}$ & $\begin{array}{c}\text { correlations } \\
\text { with Facebook } \\
\text { intrusion }\end{array}$ \\
\hline Ecuador & $N$ & $M$ & $S D$ & Pearson's $r$ & $M$ & $S D$ & Pearson's $r$ \\
Malaysia & 252 & 6.57 & 3.76 & .066 & 5.26 & 3.30 & $.143^{*}$ \\
Poland & 399 & 3.15 & 6.24 & .104 & 11.09 & 6.84 & .110 \\
Slovakia & 261 & 5.37 & 3.97 & $.231^{* * *}$ & 3.43 & 2.81 & $.217^{* * *}$ \\
Syria & 475 & 4.98 & 2.70 & $.204^{* * *}$ & 5.28 & 3.80 & $.283^{* * *}$ \\
\hline
\end{tabular}

Note. ${ }^{*} p<.05 ; * * p<.01 ; * * * p<.001$

\section{Mediation Analyses}

The analyses explored whether or not age was a moderator of the relationship between friendship as a predictor and Facebook intrusion as the dependent variable, mediated by loneliness (Figure 1). We applied a moderated mediation model. The analyses were performed by means of the program PROCESS by Hayes (2013); Model no. 59 was applied. We performed the analyses for the total sample as well as for each of the five countries separately.

To answer the question about the role of loneliness in the relation between friendship and
Facebook intrusion, we performed a mediation analyses with friendship as the predictor, loneliness as the mediator, and Facebook intrusion as the dependent variable. The analyses were performed for the total sample as well as for each of the countries. In Table 7, the mediation for total sample is presented. We used the bootstrapping method with bias-corrected confidence estimates (Preacher \& Hayes, 2004).

In the next step, we performed detailed analyses of significant interactions (Table 8). First, we analyzed the relations between friendship and loneliness at three levels of age.

As can be seen in Table 8, in the total sample and in Syria the relationships between friend-

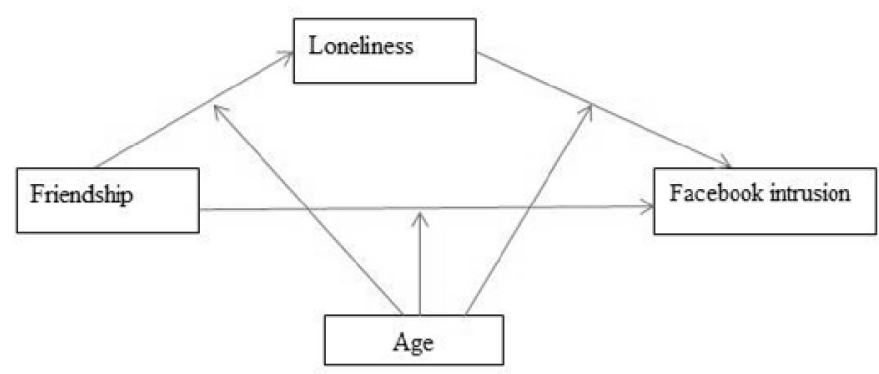

Figure 1 Conceptual model 
Table 7 Mediation analyses moderated by age (Predictor: friendship; Mediator: loneliness; Dependent Variable: Facebook intrusion)

\begin{tabular}{|c|c|c|c|c|c|c|}
\hline \multirow[b]{2}{*}{ Sample } & \multicolumn{2}{|c|}{$\begin{array}{l}\text { friendship } \\
\text { loneliness }\end{array}$} & \multicolumn{2}{|c|}{$\begin{array}{l}\text { loneliness }> \\
\text { Fbintrusion }\end{array}$} & \multicolumn{2}{|c|}{$\begin{array}{l}\text { friendship }> \\
\text { Fbintrusion }\end{array}$} \\
\hline & Coeff & $p$ & Coeff & $p$ & Coeff & $p$ \\
\hline Total sample & .007 & .027 & .005 & .582 & -.009 & .244 \\
\hline Ecuador & .001 & .911 & -.003 & .887 & .006 & .670 \\
\hline Malaysia & -.025 & .201 & -.208 & .006 & -.074 & .153 \\
\hline Poland & .003 & .665 & .025 & .350 & .025 & .380 \\
\hline Slovakia & -.007 & .288 & .012 & .582 & .002 & .931 \\
\hline Syria & .018 & .005 & .004 & .762 & -.009 & .550 \\
\hline
\end{tabular}

Note. The coefficients (Coeff) and their $p$ values refer to the interactions of a given path with age.

Table 8 Detailed analyses for significant interactions: Relations between friendship and loneliness at three levels of age in total and Syrian samples

\begin{tabular}{lccccc}
\hline Age & Effect & $t$ & $p$ & LLCI & ULCI \\
\hline Total sample & & & & & \\
$1 S D$ below mean & -.13 & -27.65 & $<.001$ & -.14 & -.12 \\
Mean & -.12 & -37.29 & $<.001$ & -.13 & -.12 \\
$1 S D$ above mean & -.11 & -23.66 & $<.001$ & -.12 & -.10 \\
\hline Syria & & & & & \\
Age & Effect & $t$ & $p$ & LLCI & ULCI \\
$1 S D$ below mean & -.15 & -15.06 & $<.001$ & -.17 & -.13 \\
Mean & -.13 & -18.44 & $<.001$ & -.14 & -.11 \\
$1 S D$ above mean & -.11 & -10.57 & $<.001$ & -.13 & -.09 \\
\hline
\end{tabular}

ship and loneliness were significant (and negative) at all age levels. Given the significant interaction, we can state that the negative relationship between friendship and loneliness was the strongest in the group of young people, somewhat less strong in medium-aged people, and the weakest (though still significant) in the oldest participants. However, the differences between age groups as regards the effect size of the relationships between the investigated variables were rather small.

Next, we analyzed the relationships between loneliness and Facebook intrusion at three age levels in Malaysia (Table 9). The relationship between loneliness and Facebook intrusion was negatively significant only in the oldest group of participants.
In Poland, the indirect effect was statistically significant (bootstrap CIs not including zero) in the sample of young people, but not mediumaged or older. In contrast, in Malaysia the indirect effect was only significant in the oldest group. Loneliness mediated the relation between friendship and Facebook intrusion in two cases: in young people in Poland and among the oldest participants in Malaysia (see Table 10).

In the next step we repeated the mediation analyses with friendship as the predictor, loneliness as the mediator, and Facebook intrusion as the dependent variable - without age in the model and with the sample consisting only of participants under the age of 30 (Table 11).

As we can see in Table 11, among young people in the Polish sample, friendship de- 
Table 9 Detailed analyses for significant interactions: Relationships between loneliness and Facebook intrusion at three levels of age in the Malaysian sample

\begin{tabular}{lccccc}
\hline Age & Effect & $t$ & $p$ & LLCI & ULCI \\
\hline $1 S D$ below mean & .16 & 0.75 & .452 & -.26 & .59 \\
Mean & -.23 & -1.70 & .090 & -.49 & .04 \\
$1 S D$ above mean & -.61 & -3.17 & .002 & -.99 & -.23 \\
\hline
\end{tabular}

Table 10 Mediation analyses moderated by age: Conditional indirect effects of friendship on Facebook intrusion at three values of age: 1 SD below mean, mean, 1 SD above mean

\begin{tabular}{|c|c|c|c|c|}
\hline & \multirow[b]{2}{*}{ Mediation at levels of age } & \multirow[b]{2}{*}{$\begin{array}{l}\text { Effect for } \\
\text { mediation }\end{array}$} & \multicolumn{2}{|c|}{$95 \% \mathrm{CI}$} \\
\hline & & & Lower & Higher \\
\hline \multirow{3}{*}{ Total sample } & $1 S D$ below mean & -.023 & -.111 & .067 \\
\hline & Mean & -.038 & -.104 & .025 \\
\hline & $1 S D$ above mean & -.05 & -.135 & .029 \\
\hline \multirow{3}{*}{ Ecuador } & $1 S D$ below mean & -.163 & -.306 & -.045 \\
\hline & Mean & -.156 & -.255 & -.061 \\
\hline & $1 S D$ above mean & -.148 & -.292 & -.024 \\
\hline \multirow{3}{*}{ Malaysia } & $1 S D$ below mean & -.118 & -.335 & .021 \\
\hline & Mean & .042 & -.071 & .162 \\
\hline & $1 S D$ above mean & .261 & .008 & .483 \\
\hline \multirow{3}{*}{ Poland } & $1 S D$ below mean & .282 & .036 & .574 \\
\hline & Mean & .175 & -.031 & .372 \\
\hline & $1 S D$ above mean & .072 & -.233 & .41 \\
\hline \multirow{3}{*}{ Slovakia } & $1 S D$ below mean & .002 & -.311 & .315 \\
\hline & Mean & .014 & -.222 & .249 \\
\hline & $1 S D$ above mean & .025 & -.358 & .409 \\
\hline \multirow{3}{*}{ Syria } & $1 S D$ below mean & -.092 & -.291 & .106 \\
\hline & Mean & -.095 & -.218 & .02 \\
\hline & $1 S D$ above mean & -.093 & -.224 & .027 \\
\hline
\end{tabular}

Table 11 Mediation in the group below the age of 30

\begin{tabular}{|c|c|c|c|c|c|c|c|c|c|c|c|}
\hline \multirow[t]{2}{*}{ Sample $(N)$} & \multicolumn{2}{|c|}{$X>M$} & \multicolumn{2}{|c|}{$\mathrm{M}>\mathrm{Y}$} & \multicolumn{2}{|c|}{$\begin{array}{l}\text { Direct } \\
X>Y\end{array}$} & \multicolumn{2}{|c|}{ Total } & \multicolumn{3}{|c|}{$\begin{array}{l}\text { Indirect } \\
X>Y\end{array}$} \\
\hline & $\mathrm{bMX}$ & pMX & bYM & pYM & bYX & pYX & $\mathrm{bT}$ & pT & b'YX & p'YX & $95 \% \mathrm{CI}$ \\
\hline Total (1539) & -.124 & $<.001$ & .040 & .461 & -.012 & .202 & -.017 & .014 & -.005 & .461 & $-.019 ; .008$ \\
\hline Ecuador (295) & -.075 & $<.001$ & .462 & .001 & .026 & .211 & -.008 & .652 & -.035 & .002 & $-.057 ;-.015$ \\
\hline Malaysia (236) & -.074 & $<.001$ & -.124 & .409 & .043 & .064 & .052 & .011 & .009 & .414 & $-.015 ; .035$ \\
\hline Poland (385) & -.170 & $<.001$ & -.242 & .033 & -.061 & .012 & -.020 & .178 & .041 & .033 & $.003 ; .082$ \\
\hline Slovakia (214) & -.124 & $<.001$ & .187 & .161 & -.002 & .924 & -.026 & .170 & -.023 & .163 & $-.059 ; .013$ \\
\hline Syria (409) & -.139 & $<.001$ & .125 & .220 & .004 & .842 & -.013 & .361 & -.017 & .221 & $-.049 ; .013$ \\
\hline
\end{tabular}


creases loneliness and loneliness decreases Facebook intrusion, whereas in Ecuador friendship decreases loneliness and loneliness increases Facebook intrusion.

\section{Discussion}

The main aim of our study was to examine the mediating role of loneliness in the relation between friendship and Facebook intrusion among five countries that differ in terms of industrial, economic, societal, and technological development: Poland, Slovakia, Syria, Ecuador, and Malaysia. Moreover, we asked questions about Facebook use, such as log hours on weekdays and weekends.

In Syria and in Poland, therelationship between friendship and loneliness is negative in each of the age groups. This relationship is the strongest among young people and the weakest in the oldest group. It seems quite logical that people who have more friends feel less lonely. As the body of studies showed, friendships provides individuals with a sense of safety, security, and support (e.g., Lyubomirsky, King, \& Diener, 2005; Hawthorne \& Griffith, 2000). It has also been shown that friendship quality is a negative predictor of school loneliness (Zhang et al., 2015). In Malaysia, the relationship between loneliness and Facebook intrusion is negative only in the oldest group of people. Older people who feel lonely rarely use Facebook in an excessive way. They do not look for friends on Facebook. However, other studies indicate that Facebook may be a platform for social contacts at all ages (Grieve \&Kemp, 2015; Sinclair \& Grieve, 2017).

Furthermore, the results of our study show that loneliness mediates the relation between friendship and Facebook intrusion among older people in Malaysia: the higher the level of friendship, the lower level of loneliness, and the higher level loneliness, the lower level of Facebook intrusion. In this country, the people who have more friends feel less lonely and the people who feel lonely score lower on the Facebook Intrusion scale. The people who feel lonely probably do not look for friends on Facebook. In limited analyses, performed only for young people under the age of 30, we found that in the Polish sample friendship decreased loneliness and loneliness decreased Facebook intrusion. Polish young people who feel lonely do not look for friends on Facebook. By contrast, in Ecuador friendship decreases loneliness and loneliness increases Facebook intrusion, which might be related with the various internet access/penetration in these countries. Ecuadorians, as the typical collective society, probably compensate for the lack of friends with Facebook activity, due to more emotional than information based use of internet communicators (Ji et al., 2010).

Our findings show a similar tendency as the study examining the relationship between Internet use and loneliness conducted by Moody (2001), who applied Weiss's (1973) bimodal theory of loneliness. The study revealed that those who scored low on both dimensions of loneliness - emotional and social - had a wide network of face-to-face friends. Internet use was negatively related to social loneliness and positively to emotional loneliness. Another study in which types of loneliness were differentiated showed that Facebook users scored higher on family loneliness, whereas Facebook nonusers scored higher on social loneliness (Ryan \& Xenos, 2011). Frison and Eggermont (2015) found that lonely people used Facebook in a more passive way.

In Poland, Slovakia, and Syria there are strong positive correlations between Facebook intrusion and $\log$ hours during weekdays and weekends. In those countries, people who have problems using Facebook in an excessive way use it every day on both working and nonworking days. It is in line with previous studies showing that Facebook Intensity was a positive predictor of Facebook addiction (Błachnio, 
Przepiorka, \& Hawi, 2016). Conversely, in Malaysia Facebook intrusion is not linked with log hours during weekdays. Malaysia has high smartphones penetration $(65 \%)$, whereas this indicator for Poland is $41 \%$ (Pew Research Center, 2015). Probably, in Malaysia a large number of people - not only those showing symptoms of Facebook intrusion but also normal users have access to Facebook not only via their computers but also via mobile devices. In Ecuador (with a relatively high rate of $60.3 \%$ of Facebook users in June 2016) ${ }^{5}$, Facebook intrusion is related to $\log$ hours only during weekends. This is quite different in comparison to the other four countries, where Facebook is used more on weekends than weekdays. As far as the regular working time is concerned, in Ecuador Facebook use is much higher than it is during weekend leisure time. The explanation can be twofold. Firstly, this might stem from the work culture customs in Ecuador, where it is quite common to use Facebook at work. Secondly, Ecuador as a highly collectivistic country, it scored only 7 on the individualism-collectivism dimension, while Poland scored 60, Slovakia 52, and Syria $35^{6}$. The collective family culture is so strong there that during the weekend time is mainly devoted to "off-line" relations with family members, not to Facebook interactions (Liddy, Hunter, Mihan, \& Keely, 2017). On the other hand, the increased Facebook use during the week might be a compensation strategy for the private contacts missing at work.

\section{Limitations and Future Research}

A limitation of the present study may be the use of self-report methods and correlational or

\footnotetext{
${ }^{5}$ Ecuador - Telecoms, Mobile, Broadband and Digital Media - Statistics and Analyses available at: http:/ /www.researchandmarkets.com/reports/838317/, retrieved June 2, 2017.

${ }^{6}$ https://www.hofstede-insights.com
}

cross-sectional designs, which have been addressed by some scholars to have limited inferential ability to prove causality. Thus, it is recommended to conduct further investigations for the present model using a longitudinal design (Bassam Mahmoud \& Reisel, 2014). The next possible limitation is the low reliability of the Friendship Scale in Malaysia and Ecuador and the Loneliness Scale in Ecuador. In future studies, these reliability indices should be increased. Another limitation was the unequal number of participants from different countries and their unequal distribution in terms of age and gender. More research is also needed to provide a better understanding of the cultural aspects of Facebook intrusion. In future research, it would be a good idea to differentiate types of loneliness, or to distinguish its social and emotional levels in accordance with the theory of loneliness proposed by Weiss (1973). The pattern of Facebook use should also be controlled, as different patterns may have different impact on mental health (Frison \& Eggermont, 2016; Hanna, Ward, Seabrook, Jerald, Reed, Giaccardi, \& Lippman, 2017), which is particularly important for friendship and the quality of relationships. The study offers some insight, which may help Facebook users improve their usage habits. The results may be useful in therapy for people with problems stemming from the excessive use of social media.

\section{Conclusion}

To conclude, the presented studies help to better understand the cultural nature of Facebook intrusion. Our results extend the knowledge about the role of friendship and loneliness in Facebook intrusion and their different degrees of dependence on culture and age. The differences between countries suggest we cannot generalize the results, and that in exploring the Facebook intrusion phenomenon culture should always be taken into consideration. 


\section{References}

Andreassen, C. S., \& Pallesen, S. (2014). Social network site addiction - an overview. Current Pharmaceutical Design, 20(25), 4053-4061.

Bassam Mahmoud, A., \& Reisel, W. D. (2014). Relating patient satisfaction to nurses' job satisfaction, job security, and obedience OCBs. International Journal of Pharmaceutical and Healthcare Marketing, 8(1), 47-61.

Błachnio, A., Przepiorka, A., Bałakier, E., \& Boruch, W. (2016). Who discloses the most on Facebook? Computers in Human Behavior, 55, 664-667. http:/ /doi.org/10.1016/j.chb.2015.10.007

Błachnio, A., Przepiorka, A., Benvenuti, M., Cannata, D., Ciobanu, A. M., Senol-Durak, E., ... Ben-Ezra, M. (2016a). An international perspective on Facebook intrusion. Psychiatry Research, 242, 385387. http://doi.org/10.1016/j.psychres.2016.06.015

Błachnio, A., Przepiorka, A., Benvenuti, M., Cannata, D., Ciobanu, A. M., Senol-Durak, E., ... Ben-Ezra, M. (2016b). Cultural and personality predictors of Facebook intrusion: A cross-cultural study. Frontiers in Psychology, 7(February 2004), 1895. http:// doi.org/10.3389/fpsyg.2016.01895

Błachnio, A., Przepiorka, A., Boruch, W., \& Bałakier, E. (2016). Self-presentation styles, privacy, and loneliness as predictors of Facebook use in young people. Personality and Individual Differences, 94, 26-31. http://doi.org/10.1016/j.paid.2015.12.051

Chopik, W. J. (2016). The benefits of social technology use among older adults are mediated by reduced loneliness. Cyberpsychology, Behavior, and Social Networking, 19(9), 551-556. http://doi.org/10.1089/ cyber.2016.0151

Elphinston, R. A., \& Noller, P. (2011). Time to face it! Facebook intrusion and the implications for romantic jealousy and relationship satisfaction. Cyberpsychology, Behavior and Social Networking, 14(11), 631-635. http://doi.org/10.1089/cyber. 2010.0318
Hawthorne, G., \& Griffith, P. (2000). The Friendship Scale: Development and properties. Centre for Health Program Evaluation Melbourne.

Liddy, C., Hunter, Z., Mihan, A., \& Keely, E. (2017). Use of Facebook as part of a social media strategy for patient engagement. Canadian Family Physician, 63(3), 251-252.

Lyubomirsky, S., King, L., \& Diener, E. (2005). The benefits of frequent positive affect: Does happiness lead to success? Psychological Bulletin, 131(6), 803855. http://doi.org/10.1037/0033-2909.131.6.803

Özdemir, Y., Kuzucu, Y., \& Ak, Ș. (2014). Depression, loneliness and Internet addiction: How important is low self-control? Computers in Human Behavior, 34, 284-290. http://doi.org/10.1016/j.chb.2014. 02.009

Petrocchi, N., Asnaani, A., Martinez, A. P., Nadkarni, A., \& Hofmann, S. G. (2015). Differences between people who use only Facebook and those who use Facebook plus Twitter. International Journal of Human-Computer Interaction, 31(2), 157-165. http://doi.org/10.1080/10447318.2014.986640

Rosseel, Y. (2012). lavaan: An R Package for Structural Equation. Journal of Statistical Software, $48(2)$.

Song, H., Zmyslinski-Seelig, A., Kim, J., Drent, A., Victor, A., Omori, K., \& Allen, M. (2014). Does Facebook make you lonely?: A meta analysis. Computers in Human Behavior, 36, 446-452. http:// doi.org/10.1016/j.chb.2014.04.011

Wegmann, E., \& Brand, M. (2016). Internet-Communication Disorder: It's a matter of social aspects, coping, and internet-use expectancies. Frontiers in Psychology, 7(November), 1-13. http://doi.org/ 10.3389/fpsyg.2016.01747

Weiss, R. S. (1973). Loneliness: The experience of emotional and social isolation. Cambridge, MA, US: The MIT Press.

Zhang, X., Chen, F., Wu, Y., Yuan, C., \& Wang, Y. (2015). Examining relations between locus of control, loneliness, subjective well-being, and preference for online social interaction. Chinese Journal of Clinical Psychology, 23(1), 147-149. 